\title{
COMPORTAMIENTO AGRONÓMICO Y CALIDAD \\ DEL GRANO DE SIETE CULTIVARES DE CAFÉ MANEJADO BAJO SOMBRA, MASATEPE, NICARAGUA
}

\author{
Aurora del Carmen Suárez Rivera ${ }^{1}$, Jaime Ricardo Picado Aragón²; Rodolfo Murguía Hernández³; Jeremy Haggar ${ }^{4}$ \\ ${ }^{1}$ Instituto Nacional de Tecnología Agropecuaria (INTA) \\ ${ }^{2}$ Certificadora BIOLATINA, Nicaragua \\ ${ }^{3}$ MSc. Universidad Nacional Agraria. Managua, Nicaragua \\ 4. Centro Agronómico Tropical de Investigación y Educación
}

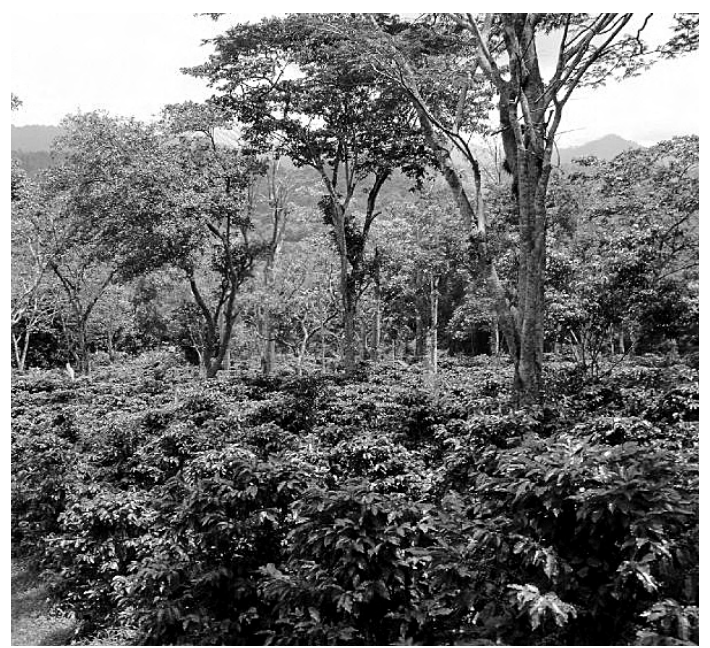

\section{RESUMEN}

Fueron establecidos y manejados desde el 2000, las variedades de café Catrenic, Catrenic injertada sobre Robusta (CARO), Pacas, pacas injertadas en Robusta (PARO); adicionalmente se valoraron tres líneas híbridos de café L2,A30; L3,A17; L.13, A.12, conocidos como híbridos 2,3 y 4 respectivamente, los que se establecieron en un diseño de bloques completos al azar. Se analizaron las variables número de frutos contenidos en un $\mathrm{kg}$ de peso, peso pro-medio de frutos, la producción de grano oro, análisis físico de los granos (cribas) y la calidad de taza (acidez y cuerpo); fue valorado el tipo de café y clasificado según la norma técnica nicaragüense (NTON 03-025-03) y el respectivo análisis de mercado. Se obtuvo que Catrenic requiere de menor cantidad de grano por $\mathrm{kg}$ de peso por mayor peso del grano y grosor. Todas las variedades mejoraron en el ciclo productivo de mayor precipitación. En relación a la producción de grano oro, el Hibrido 2 (L2,A30) manifestó el mayor incremento en el tiempo. El tamaño de los granos mejoró producto de las condiciones de humedad que prevalecieron y favorables para Pacas, y para PARO. Tanto el análisis de acidez y cuerpo fue variable en las muestras, sin embargo, los híbridos 2 y 4 mostraron un mayor por ciento en la calificación de Bueno. Excepto en el ciclo 2005 / 2006 los híbridos 2, 3 y 4 y Catrenic y CARO, resultaron Bueno entre un 20 a un $60 \%$, coincidiendo con las mejores condiciones de precipitación. En los ciclos 2005 / 2006 y 2006 / 2007 en todas las variedades fue calificado el café en la categoría BB, que en ese momento el precio establecido era de US \$ 134.42 el quintal oro.

\section{ABSTRACT}

Four coffee varieties (CATRENIC, CARO, PACAS y PARO were planted in year 2000. Additionally three hybrids were tested L2,A30; L3, A17; L.13, A.12 known along the text as Hibrid 2, 3 and 4 respectively. The four varieties and the three hybrids were arranged in a randomized Block Design (BCA). The variables number of coffee berries per kilogram, mean weight of each coffee berries, green coffee production, quality of the green coffee (size), fresh coffee cup (acidity and body test) were measured during this study. The nicaraguan technical norm (NTON) for coffee market was considered for coffee classification. CATRENIC coffee variety needs less number of coffee berries to obtain one kilogram of green coffee. The hybrids 2 and 4 presented higher acidity content and were classified as good. From 2005 to 2007 the coffee was classified as BB because the climate conditions and the coffee price has a mean of 134.42 American dollars per $45 \mathrm{~kg}$ In general the higher amount of precipitation in some years favours de yield and quality of the four coffee varieties and the three coffee hybrids evaluated in the pacific area of Nicaragua. 
$\mathrm{E}$ n Nicaragua, se cultivan unas 130680.9 ha. de café bajo sombra (ORGANICOOP, 2007); mientras que las exportaciones totales del país representaron el $19.54 \%$ equivalentes a 69718 600 US \$ correspondiente al ciclo agrícola $2001-2002$, aumentando a 281991061.75 US \$ en el ciclo 2007 - 2008 (CETREX, 2008), originado por una mejora de los precios internacionales. Las calidad, productividad, resistencia a plagas y enfermedades y la capacidad de una amplia adaptación deben estar presentes en los futuros cultivares de café (Fischersworring y Robkamp, 2001; Van Hintum et al., 2003), donde las actuales variedades en Latinoamérica no las presentan (Bertrand et al., 1999) en particular las variedades de Coffea arábica en América Central, por que provienen de una base genética muy estrecha (Bertrand y Anthony 1995; Anthony et. Al., 2003).

Tomando en cuenta esa limitada variabilidad genética y los problemas que ello significa en los procesos de mejoramiento genético, el CATIE, Costa Rica, inició en los años 50 la utilización de recursos genéticos, que más tarde en 1994 establecen el Programa de Mejoramiento de Café (PROMECAFE), en $C$. arábica y la creación de una nueva base genética en colaboración con la Cooperación Francesa a través del Centro para la Cooperación Internacional en la Investigación Agrícola para el Desarrollo (CIRAD). El programa ha recolectado en Etiopia, Sudan, Kenia y Tanzania en los años 1964 y 1966 para aumentar la base genética del material y conservados en el banco de germoplasma en CATIE; posteriormente los estudios han identificado los individuos con cualidades agronómicas de mayor interés para el mejoramiento genético, e iniciaron cruzamientos con las variedades comercialmente cultivadas obteniéndose familias de híbrido F1 (Bertrand et al., 2003).

Actualmente se evalúan en la región Centroamericana híbrido $\mathrm{F} 1$, los que muestran superioridad a las variedades tradicionales en producción, calidad bajo diferentes condiciones agroecológicas.

Por lo anteriormente expuesto, el presente estudio tuvo el propósito de evaluar el comportamiento agronómico y de calidad física, organoléptica de grano y de mercado de siete genotipos de Coffea arabica L.

\section{MATERIALES Y MÉTODOS}

El experimento fue establecido con dos réplicas en áreas del Centro de Capacitación y Servicio Regional del Pacifico de Nicaragua ( $\left.11^{\circ} 54^{\prime} \mathrm{LN}, 86^{\circ} 09^{\prime} \mathrm{LW}\right)$ hoy Centro Nacional de capacitación CENACOOP, CARUNA, a una altitud de $455 \mathrm{msnm}$, precipitación anual de $1400 \mathrm{~mm}$ y temperaturas de $24^{\circ} \mathrm{C}$ y humedad relativa entre $70-80 \%$, (Herrera, 2000). Una tercera réplica fue establecida en áreas del Centro Experimental de Campo Azules (12 $\left.12^{\prime} \mathrm{LN}, 86^{\circ} 04^{\prime} \mathrm{LW}\right)$ propiedad del Instituto Nicaragüense de Tecnología agropecuaria (INTA), a una altitud de $474 \mathrm{msnm}$; en el Municipio de Masatepe, departamento de Masaya.

Los cultivares de café se encuentran establecidos bajo sombra de combinaciones arbóreas como: Inga laurina (Sw.) Willd + Simarouba glauca DC; Samanea Saman (Jacq.) Merr + Tabebuia rosea (Bertol.) DC., a dichas parcelas de manera separada se aplican niveles de insumos orgánico moderado $(\mathrm{OM})$ y convencional moderado (CM); sin embargo por las características de tener un insuficiente número de repeticiones para sombra e insumos solo fue considerado el análisis del factor variedad (Tabla 1). El cafe fue plantado a $2 \mathrm{~m}$ entre surcos y $1.25 \mathrm{~m}$ entre plantas para obtener 4000 plantas por hectárea.

Para llevar a cabo el estudio se tomaron mediciones de las siguientes variables:

Tabla 1. Variedades evaluadas en el municipio de Masatepe, Masaya. 2006 - 2008

\begin{tabular}{|c|c|c|c|}
\hline No. & Tratamientos & Línea & Origen \\
\hline 1 & Catrenic & Catrenic & $\begin{array}{l}\text { HW26.19/1 } \\
\text { CATURRA ROJO }\end{array}$ \\
\hline 2 & Caro & & $\begin{array}{l}\text { Catrenic injerta en } \\
\text { Robusta }\end{array}$ \\
\hline 3 & Híbrido 2 & $\mathrm{~L} 2, \mathrm{~A} 3 \mathrm{O}$ & Caturra 9*ET 15 \\
\hline 4 & Híbrido 3 & L3, A17 & Caturra * ET 15 \\
\hline 5 & Híbrido 4 & L.13 A.12 & T 5296*ET6 \\
\hline 6 & Pacas & Pacas & Mutación de Bourbón \\
\hline 7 & Paro & & $\begin{array}{l}\text { Paca injertas sobre } \\
\text { Robusta }\end{array}$ \\
\hline
\end{tabular}

Nota: Se utilizaron las claves de las variedades en el texto.

Componentes del rendimiento y calidad de grano Número de frutos por kg (Nfrukg). De un kilogramo de café uva por tratamiento y parcela, se contabilizaron el número de frutos que forman dicho peso.

Peso del fruto en gramos (Pfrug). Se obtiene a partir de la relación del peso de un $\mathrm{kg}$ de frutos maduros entre el número de frutos que compone el $\mathrm{kg}$ por parcela $\mathrm{y}$ variedad. 
Producción de grano (kg) oro por ha (kgoroha). De la relación obtenida entre grano uva a grano oro, se procedió a la obtención del rendimiento por unidad de área por cada tratamiento en estudio. El café después de cortado, fue despulpado, fermentado, lavado, secado y trillado para su almacenamiento.

\section{Análisis físico, organoléptico del grano y de} mercado

Cribado del grano: Cada muestra de café por parcela y variedad fueron tamizados o cribados a diferentes tamaños en orden descendente, tales como cribas 20 , $19,18,17,16,15$ y 14 .

Calidad de taza: La catación fue realizada por personas entrenadas y especializadas que determinan las características organolépticas, como acidez y cuerpo, siendo de carácter cualitativo y cuantitativo, al cual dan una valoración.

Análisis de Tipo de café: Es la clasificación de café verde, a partir de la norma técnica NTON 03025 - 03; donde el producto café se clasifica de acuerdo a las características desarrolladas después del procesamiento de beneficiado húmedo, beneficiado seco, y sus atributos físicos y sensoriales del producto final.

Tipo mercado: El análisis de mercado se refiere a los porcentajes del total de las muestras por variedad, determinado por sus características físicas y organolépticas a un mercado específico de calidad, con distintas categorías (AAA, AA, A, B, BB, C 0, C 1, C 2, C 3 y C 4); esta clasificación es la utilizada por el Centro de trámites de las exportaciones (CETREX, 2008).

Análisis de datos. A las variables de crecimiento y desarrollo y rendimiento se aplicó análisis de varianza (ANDEVA) para experimento unifactorial y las medias de los tratamientos se les sometió a la prueba de separación de medias por LSD a una probabilidad del $95 \%$ de confianza; utilizándose el programa estadístico SAS, (2003).

\section{Producción de grano}

\section{RESULTADOS Y DISCUSIÓN}

Número de frutos por kilogramo (Nfrukg). Durante la tercera cosecha los híbridos, necesitaron mayor número de frutos para componer un kg de café uva, esto se aduce a un menor peso del grano, también se obtuvo que la variedad Caro (Caturra injertada en Robusta) y café uva para conformar un $\mathrm{kg}$ y se debe a la presencia de un grano con mayor tamaño, no diferenciándose estadísticamente (Prob $>$ F: 0.3428). En la cuarta cosecha, todas las variedades muestran un mejor comportamiento, en el número de frutos para conformar un kilogramo uva, Catrenic presenta una menor relación del número de frutos para conformar un $\mathrm{kg}$ uva, siendo los Híbrido 2, 3 y 4 necesitaron para esta cosecha un mayor número de frutos para formar un $\mathrm{kg}$ uva $(\mathrm{P}>\mathrm{F}=$ 0.013 2) como se aprecia en la Figura 1.

Para la quinta cosecha no se observaron diferencias estadísticas $(\mathrm{P}>\mathrm{F}=0.1851)$, en los tratamientos, sin embargo se repite la tendencia en esta cosecha para los cultivares híbridos las cuales presentan la mayor cantidad de frutos para conformar un $\mathrm{kg}$. De forma general, para las tres cosechas los híbridos, necesitaron un mayor número de frutos para ajustar un kg de café uva y las variedades comerciales en las tres cosechas requirieron un menor número de frutos para conformar un $\mathrm{kg}$. Este resultado puede estar relacionado con el aspecto adaptabilidad de las variedades comerciales a los factores ambientales del ensayo, como a la cantidad y distribución de las precipitaciones a los cuales los híbridos posiblemente se están adaptando para crear resistencia.

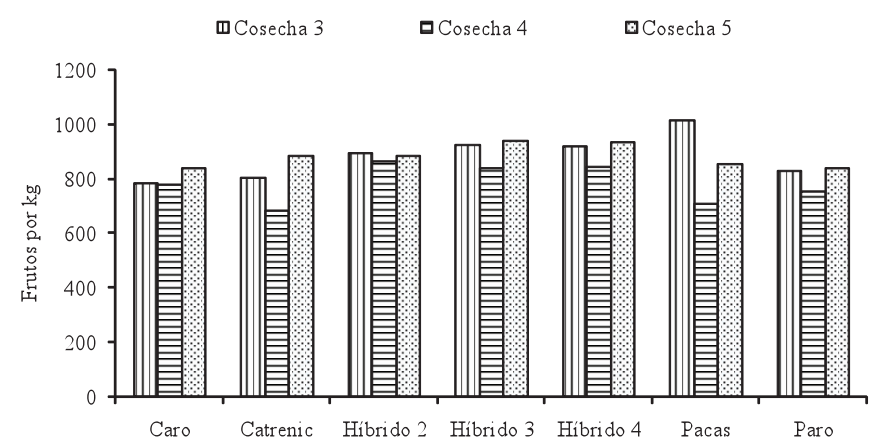

Figura 1. Número de frutos por kg (Nfrukg) de café uva en cuatro variedades y tres híbridos de café, Masatepe, Masaya. $2004-2007$.

Peso de fruto (g) (Pfrug). En los resultados obtenidos para la tercera cosecha no es estadísticamente significativa $(\mathrm{P}>\mathrm{F}=0.4638)$; sin embargo, la variedad Caro (Caturra injertada en Robusta) presenta el mayor peso en gramos, seguida de Catrenic y de Paro (Paca injertada en Robusta) con el tercer mayor peso de fruto, los híbridos presentaron una tendencia media, siendo la variedad Pacas la de menor peso (Figura 2).

En la cuarta cosecha Catrenic presenta superioridad estadística $(\mathrm{P}>\mathrm{F}=0.0243)$ ante todas las variedades; según Bolaños (2005), la variedad expresa un mayor 
tamaño de grano con relación a otras variedades en el país, esto coincide con los resultados del presente estudio en la variable tamaño de Criba, donde Catrenic presentó una mayor distribución de granos de café oro con cribas superiores a 17 en relación a las demás variedades. Pacas obtuvo el segundo mayor peso; Santoyo, et al., 1996 menciona que las variedades, Bourbon, Typica de las cuales proviene la variedad Pacas presentan un tamaño de grano medio.

Las variedades injertadas sobre Robusta CARO y PARO, expresaron menor tamaño que las mismas variedades no injertadas, esto puede atribuirse a que el patrón Robusta presenta granos de menor tamaño y menor densidad que las variedades arábigas (Santoyo et al., 1996); así mismo los Híbrido 3 e Híbrido 4, expresaron un menor tamaño, pudiendo atribuirse a que estas variedades poseen en su cruzamiento la variedad Caturra la que según Santoyo et al., (1996), presenta granos pequeños.

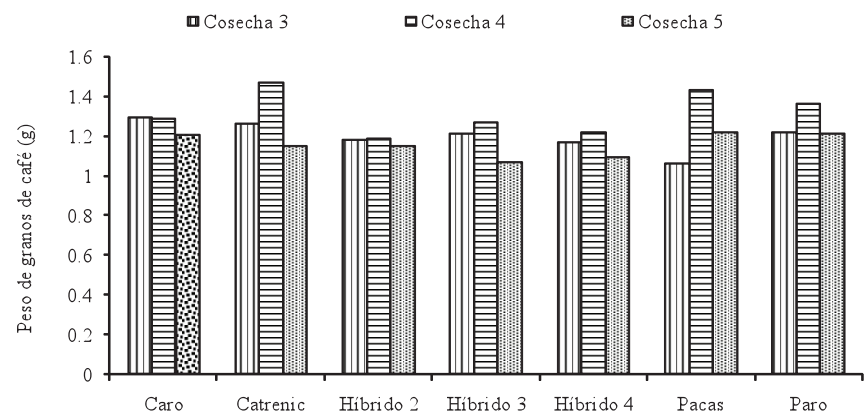

Figura 2. Peso de fruto (g) (Pfrug) durante tres cosechas en cafetos de cuatro variedades y tres híbridos de café, Masatepe, Masaya, $2004-2007$.

Producción de grano oro $\left(\mathrm{kg} \mathrm{ha}^{-1}\right)$. Se analizó la variable Producción de grano oro $\left(\mathrm{kg} \mathrm{ha}^{-1}\right)$ obtenido para todos los tratamientos durante tres cosechas, sin embargo, es posible mencionar las condiciones extraordinarias de la cosecha 4, donde alguno de los tratamientos fueron objeto de un manejo de tejido intensivo en comparación con las demás cosechas, expresando una disminución en la producción siendo entonces que entre las cosechas 3 y 5 , únicamente los materiales Híbrido 2 e Híbrido 3 presentaron un ascenso en la producción en comparación a las demás variedades, por otra parte se puede observar que el material Híbrido 2, es el que manifestó el mayor incremento en la producción en el tiempo de evaluación (Figura 3).

Durante la tercera cosecha los materiales híbridos presentaron la menor producción en comparación a las variedades comerciales y siendo estadísticamente diferentes $(\mathrm{P}>\mathrm{F}=0.1005)$, según Hidalgo (2007), una posible explicación es la precocidad productiva que se atribuye a este material, ya confirmado esto por Bertrand et al (1997) que los híbridos expresan una mayor precocidad que las líneas puras.

Un estudio realizado en Heredia, Costa Rica por Hidalgo (2007), a $1800 \mathrm{msnm}$, temperatura promedio de $20,5^{\circ} \mathrm{C}$ y $2200 \mathrm{~mm}$ de lluvia, obtuvieron resultados similares a este estudio para el hibrido 2 , el que fue superior al híbrido 4, los que mantienen una producción superior en comparación a variedades comerciales. Estos resultados son coincidentes al expresado en el presente estudio en la cosecha 5 con relación a las variedades comerciales reflejándose entonces la superioridad $(\mathrm{P}>\mathrm{F}$ $=0.1864)$, de los Híbridos 2 y 4 .

Esta característica en los híbridos con mayor rendimiento, es conferida a la expresión del vigor híbrido o heterosis originado como el resultado de la combinación e interacción de los genes paternos en la primera descendencia, esta característica ha sido reportada en el pasado por Bertrand et al., (1997) sobre la especie Coffea arábica L.

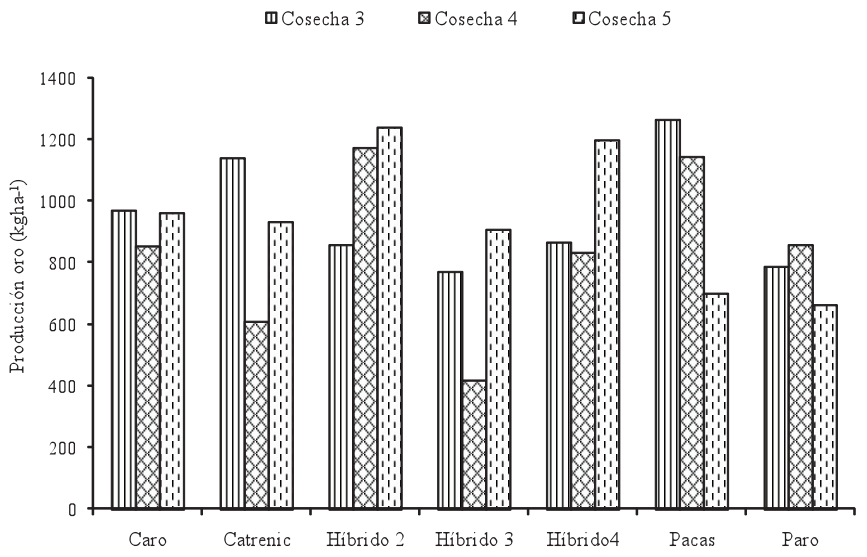

Figura 3. Producción del grano oro $\left(\mathrm{kgha}^{-1}\right)$ durante tres cosechas en cuatro variedades y tres híbridos de café, Masatepe, Masaya 2004 - 2007.

\section{Análisis Físico y organoléptico}

Análisis físico de los granos de café. El análisis de granulometría demuestra que las variedades Pacas y Paro, expresaron la mayor cantidad de granos de café con criba inferior a 15 , y Catrenic obtuvo la mejor relación de criba superior a 17, los datos coinciden con lo encontrado por Baylon y Pizzi $1994 b$ en donde Catrenic obtiene un porcentaje mayor de granos con tamaño de criba $>17$ comparados a Pacas con un menor cantidad de granos resultados que se pueden observan en la Figura 4. 
Las variedades Híbridas F1, obtuvieron la mejor relación de criba entre 15 - 17, donde Híbrido 4 y 3 presentan superioridad ante el Híbrido 2, esto coincide por lo señalado por (FONTAGRO, 2006) donde menciona que el grano de café de cultivares híbridos es similar y a veces superior al tamaño de grano de variedades tradicionales.

Los granos de café de las cuatro variedades y tres híbridos en estudio fueron distribuidos entre $65.39 \%$ y el $78.39 \%$ con criba entre $15 / 64$ y $17 / 64$, lo que coincide con Bolaños (2008), en donde el resultado para varias variedades de café del país, (Catuai, Caturra y Pacamara), presentan el mayor porcentaje en Criba $17 / 64,16 / 64,15 / 64$ para un $74.87 \%$ que representan el total del tamaño de los granos, y con lo encontrado por Muschler, (1998) donde la mayor cantidad de granos encontrados de distintas variedades Catuai y Caturra en café bajo sombra se concentran es estas categorías.

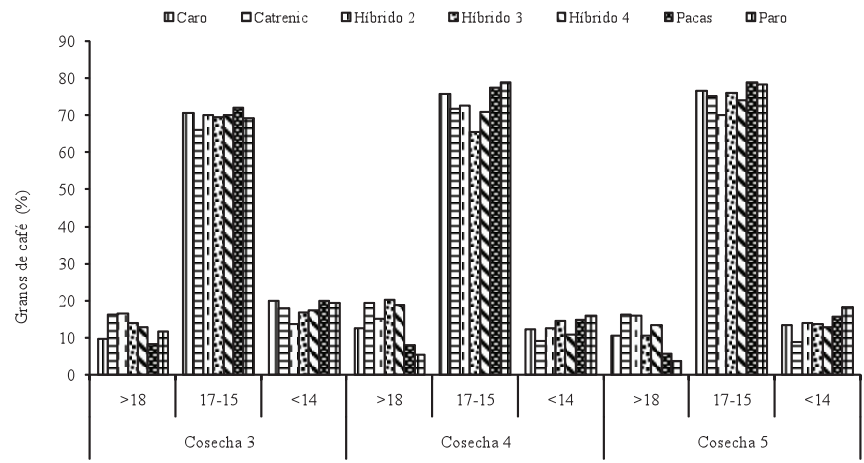

Figura 4, Análisis de criba durante tres cosechas en cuatro variedades y tres híbridos de café, Masatepe, Masaya, 2004 -2007 .

Así se logra observar durante las tres cosechas que Catrenic expresa menor tamaño de grano al estar injerta, este comportamiento es claro ya que según Santoyo et a.,, (1996), el café Robusta produce granos de menor tamaño el que puede estar influenciado a la variedad (Figura 4). Avilan (2008), indica que el patrón al proporcionar el sistema radical posee una capacidad propia que puede ser parcialmente modificada por la acción del injerto y ésta última a su vez, sufrir alteraciones por la acción del primero, estableciéndose una interacción que induce cambios en el comportamiento de los materiales que conformaron la unión.

Análisis de acidez. La acidez es la característica más apreciada en la comercialización del café, y por consiguiente, con más valor. El análisis organoléptico realizado por el catador y según su categorización (Excelente, Bueno, Regular / Bueno y Regular) que significa que posee una cantidad expresada en el atributo; esto quiere decir por ejemplo que cuando se da la categoría excelente, el catador define esto por la cantidad exagerada del atributo evaluado de acuerdo a su evaluación y ese café puede optar para mercados especiales; así la categoría Bueno que indica que presentó bastante, Regular/Buena cantidad media en comparación de los extremos y Regular es decir poco atributo calificado (Orozco, 2009: Comunicación personal). ${ }^{1}$

La acidez se ha asociado a la acción de ácidos orgánicos, entre ellos el acido fosfórico puede ser uno de los responsables de la expresión de esta característica (Clifford, 1985). Con un porcentaje no menor del 62,5\%, de todas las muestras analizas fueron catalogadas de Regular a Regular/Bueno, en donde las mejores calificaciones fueron para la variedad Pacas con un 37,5 $\%$ de las muestras como Buena durante la cosecha 3; la variedad Catrenic obtuvo el $75 \%$ de las muestras en la categoría Regular/Bueno en la cosecha 3 y un 87,5 $\%$ en las cosechas posteriores Los híbridos, presentaron porcentajes de hasta un 33,33\% de las muestras con calidad de Buena, siendo el Híbrido 2 que expresó la mejor acidez con un $25 \%$ en la cosecha 3 y un $33,33 \%$ en la cosecha 4 de calidad Buena. Figura 5.

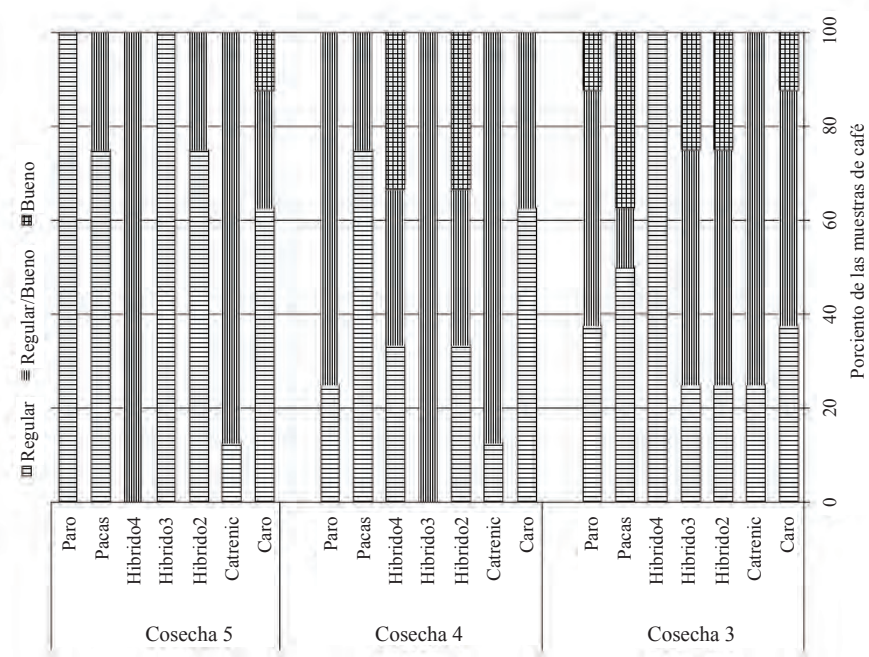

Figura.5 Análisis de acidez durante tres cosechas en cuatro variedades y tres híbridos de café en Masatepe, Masaya. 2004 -2007 .

Análisis de cuerpo. Es el último elemento de evaluación; el cuerpo se determina por la densidad o pesos de la bebida en la boca (Katzeff, 2001). El término de cuerpo es utilizado para describir la sensación del café

Orozco, J. 2009. Certificadora de Café Nicaragüense (CECAFENIC), Managua, Nicaragua. 
en la boca y por consiguiente con mejor valor (Cleves y Astúa, 1998).

Los mayores porcentajes de muestras obtenidas durante las tres cosechas fueron clasificadas en las categorías Regular y Regular/Bueno en el indicador cuerpo; sin embargo Catrenic en la cosecha 3 obtuvo una clasificación de Bueno en el 50\% de sus muestras. Mientras tanto los Híbridos 2 y 4 obtuvieron una clasificación de Bueno en el $66.7 \%$ y Paro en el $75 \%$ de sus muestras de café en la cosecha 4 (Figura 6).

En la cosecha 3, Catrenic presentó en un 50\% de las muestras obtuvo una clasificación de Bueno. Lara(2005), indica que el incremento en tamaño y peso (Figura 2 y 4), implica una mayor acumulación de materia grasa, esta acumulación de materia grasa favorece el aumento en la intensidad de las características organolépticas: aroma, acidez y cuerpo.

Los resultados encontrados por Cardoza y Jiménez (2007), bajo las mismas condiciones de sombra, obtuvieron resultados similares a la presente investigación, clasificando para las cualidades organolépticas de cuerpo los mayores porcentajes de las muestras dentro de las categorías Regular y Regular/ Bueno.

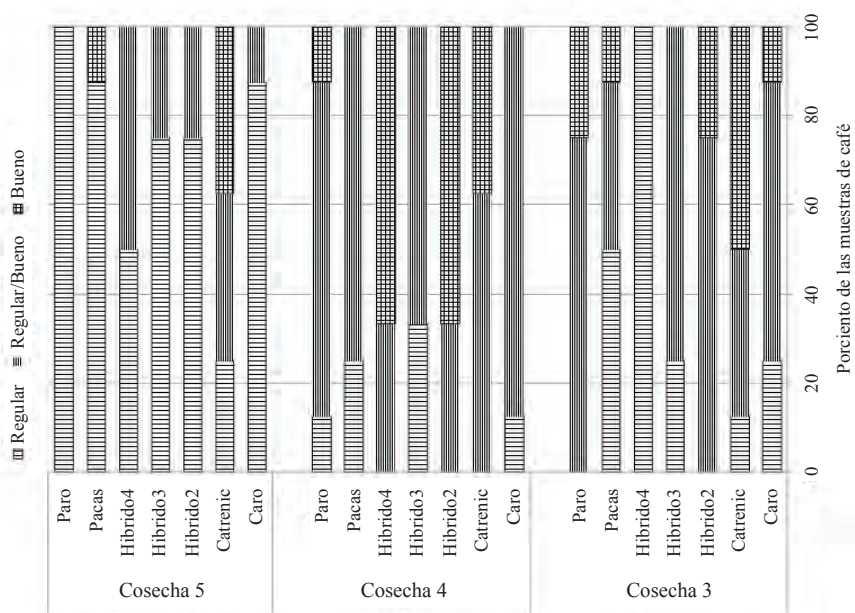

Figura 6. Análisis de cuerpo durante tres cosechas en cuatro variedades y tres híbridos de café en Masatepe, Masaya. $2004-2007$.

Tipo de café producido. Las características como el tamaño del grano, acidez, aroma, cuerpo y licor, nos dará como resultado la clasificación según la norma técnica nicaragüense, NTON 03 025-03, del tipo de café producido para café de volumen. Se puede observar de forma general que se obtuvieron dos clasificaciones Tipo Lavado Nicaragua (GW) característico de la zona del pacifico de Nicaragua, es un café básico aceptado por la bolsa de Nueva York para ser entregado o recibido en los contratos del CSCE (Centavos de dólar por libra) y Tipo Estrictamente de Altura (SHG), que se dá en altitudes superiores a los $750 \mathrm{msnm}$ este tipo de café representa el $75 \%$ de la producción cafetalera de Nicaragua que se produce en la zona norte del país (Jinotega, Matagalpa, Nueva Segovia, Madriz y Estelí), también se produce en Boaco, parte del volcán Mombacho en Granada y en la Sierras de Managua en el Crucero.

Todas las muestras resultaron ser tipo GW, excepto un $25 \%$ de la muestra del Híbrido 2 obtuvo SHG; tanto Catrenic como Híbrido 2 obtuvieron una mayor proporción con un de $62.5 \%$ y $66.67 \%$ respectivamente del tipo SHG, lo cual significa que bajo condiciones particulares dadas en el sitio de estudio se obtienen cafés similares a los producidos a mayores alturas (750 msnm) del país con una clasificación SHG, para la cosecha 4 (Figura 7).

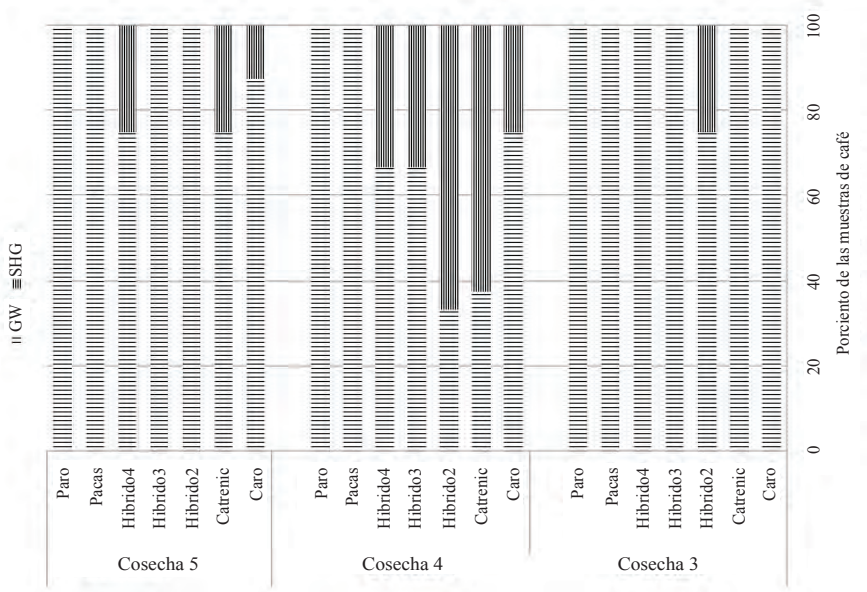

Figura 7. Análisis de tipo de café producido durante tres cosechas en cuatro variedades y tre híbridos de café, Masatepe, Masaya. 2004 - 2007-

Se observó un comportamiento particular en la cosecha 4, para todas las variedades estudiadas, el Híbrido 2 y la variedad Catrenic desarrollaron un porcentaje importante de la clasificación SHG (Tipo estrictamente de Altura), lo cual coincide con los mayores porcentajes de precipitación del año con $1819.3 \mathrm{~mm}$ anuales, este comportamiento, es reconocido por Fischersworring y Robkamp (2001), indicando que Coffea arábica L en condiciones óptimas de precipitaciones y temperatura mejora las características de sensoriales.

Análisis de mercado. La calidad del grano de café, se destaca a través de un conjunto de atributos organolépticos que son requeridos por un mercado de consumidores. 
El análisis de mercado realizado durante esta investigación, presenta la tendencia a las categorías, según su calidad, descrita por el catador, después de los análisis físico-químico, bioquímico y humedad de las muestras de los cultivares evaluados; según CETREX (2008), existen las siguientes categorías para la exportación: AAA, AA, A, B, BB, C 0, C 1, C 2, C 3 y $\mathrm{C} 4$, estas categorías están determinadas por la cantidad de defectos encontrados por muestras de $300 \mathrm{~g}$, que es analizada en laboratorio. Esta clasificación permite establecer un precio de referencia para cada una de las categorías definidas sobre la base del control de calidad según la Norma Técnica Nicaragüense -NTON de Muestreo de granos comerciales. Para la cosecha 3, en la mayoría de los genotipos logran categoría $\mathrm{B}$, mientras que un bajo porcentaje fueron clasificadas como tipo A en las variedades Caturra injerta en Robusta (Caro) y Pacas.

En el caso de las cosechas 4 y 5, las muestras mostraron los mayores porcentajes para un mercado $\mathrm{BB}$, con la excepción de la variedad Híbrido 2 (cosecha 4) con un $33.33 \%$ de las muestra y Catrenic (cosecha 5) con un $12.5 \%$ en la categoría de mercado tipo A (Figura 8). Los resultados que determinan la calidad, la calidad se refiere a los atributos del producto, que contribuyen a la satisfacción del consumidor. En café, se puede definir, libre de materiales extraños, certificado orgánico, café amigable con los pájaros, etc. (Pholan, et al., 2006). Figura 8.

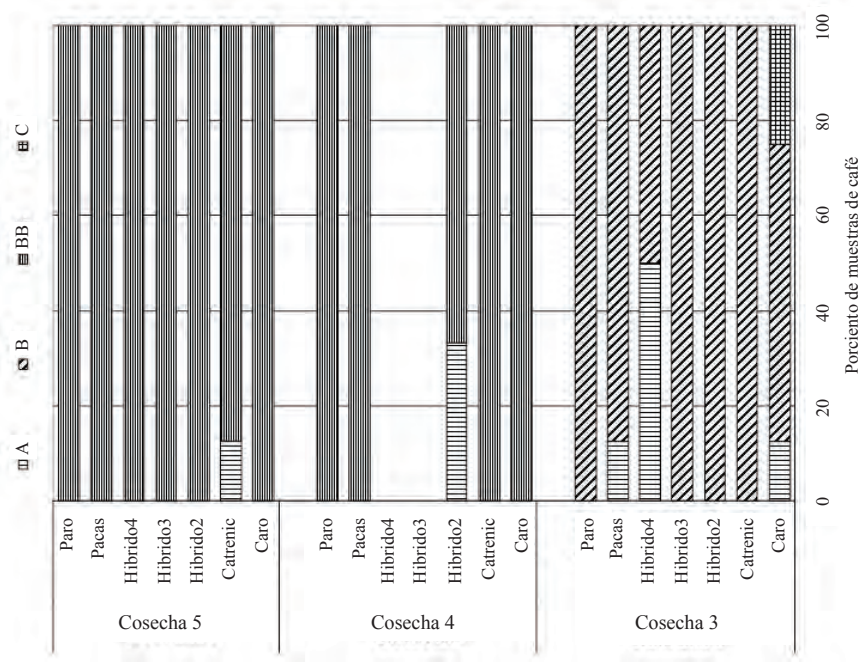

Figura 8. Análisis de mercado de calidad durante tres cosechas de cuatro variedades y tres híbridos de café, Masatepe, Masaya 2004 - 2007.
Según CETREX (2008), el café oro grano verde alcanzó precios según calidad por quintal el tipo B \$ 132.62 el tipo BB 134.42 \$ y el tipo A 141.7 \$.

\section{CONCLUSIONES}

Los frutos producidos en la cosecha 4 por la Variedad Catrenic obtuvieron el mayor peso de fruto (g) lo que implica menor cantidad de frutos por libra, superando a las variedades injertas, así como los Híbrido F1, que presentaron el menor peso.

La mayor producción de café en el tiempo fue presentado por las variedad, Híbrido 2 que fue superior a los Híbridos 3 y 4, siendo estos los únicos que presentaron un ascenso en sus rendimientos durante las cosechas en estudio, Paca presentó el segundo mejor rendimiento durante las cosechas 3 y 4 no así durante la cosecha 5, Catrenic presentó un comportamiento intermedio.

El análisis de granulometría evidencia que durante las cosechas en estudio entre $65.39 \%$ y el $78.39 \%$ de las variedades presentan criba entre 15 y 17, así las variedades Paca y Paro expresaron la mayor cantidad de granos de café con criba inferior a 15 y Catrenic obtuvo los mejores porcentajes de criba superior a 17 .

Las variedades e híbridos F1, mejoran la acidez a calidad buena en condiciones de precipitación abundante, como las ocurridas en 2004 y 2005; así como el tipo de manejo agroforestal; sin embargo, el comportamiento es muy variable en las tres cosechas.

Con relación al análisis de cuerpo, se presentó similar comportamiento influenciado por las condiciones de lluvia en la zona y el tipo de manejo agroforestal, donde se mejora de bueno/regular a bueno.

El análisis del tipo de café que se produce, se demuestra que se alcanza un mejor café como SHG influenciado por la cantidad y distribución de las precipitaciones y del sistema de manejo agroforestal.

El grano verde de café producido en la zona se podrá comercializar en el mercado exterior como tipo BB. 


\section{REFERENCIAS BIBLIOGRÁFICAS}

Anthony, F; Astorga, C; Quiros, O; Bertrand, B; Etienne, H; Topart, P; Lashermes, L. 2003. Diversidad genética de los cafés (Coffea arabica) silvestres y cultivados, revelada por marcadores moleculares. Boletín PROMECAFE no. 96.pp. 7-12.

Avilan, L. 2008. El patrón y su importancia en la fruticultura. (en línea). Caracas, Ve. Consultado, 18 de Enero.2009. Disponible en http://74.125.113.132/search?q=cache:bMdFopJsbpUJ:www.ceniap.gov.ve/ publica/fdold/fd58/patrones.html+efectos + del+patron+injerto+en + cafe\&hl=es\&gl=ni\&strip=1

Bertrand, B; Anthony F; Etienne, H. 2003. Proyecto regional de mejoramiento genético del arabica en centro América. Sintesis de las actividades junio 1 991- junio 2003. PROMECAFE-CATIE-COOPERACION FRANCESA.

; Aguilar, G; Santacreo, R y Anzueto, F. 1999. El mejoramiento genético en América Central. 1999. Desafíos de la Caficultura en Centroamérica. ed. Por Benoit Bertrand y Bruno Rapidel.- San José, C.R.: IICA. PROME-CAFÉ: Híbrido : IRD : CCCR.FRANCIA, 1999.496 p

; Anthony F. 1995. El mejoramiento genético de Coffea arábica en América Central. In Simposio HÍBRIDO/CATIE Mejoramiento genético y desarrollo de los cultivos tropicales (1995, Turrialba, CR). Resúmenes. Turrialba, CR, HÍBRIDO/CATIE.Pp32

Bolaños, M. 2005. Ensayos de sistemas de café. La calidad del café durante los primeros años del ensayo ciclo cafetalero 2004/2005. Pág. $1-7$.

Cardoza, M y Jimenez, E. 2007. Evaluación de rendimientos de café. (Coffea árabica L.) Bajo la influencia de diferentes manejos agroforestales en Masatepe, Nicaragua. Tesis Ing. Agrónomo Universidad Nacional Agraria. Managua, Nicaragua. $54 \mathrm{pp}$

CETREX. (Centro de trámites de exportaciones, Ni). 2008. Estadísticas de Exportaciones de Café Verde (en línea). Consultado el 10 oct. del 2008. Disponible en http:/www.cetrex.com.ni/cafe.html

Cleves, R; Astua, R. 1998. Defectos y vicios del café que se originan o manifiestan en el beneficiado. In Cleves, S. Eds. Tecnología en beneficiado de café, San José, C, R. P 1521

Fischersworring Y .H, Robkamp. R. 2001 Guía para la Caficultura Ecológica/ Primera Edición/ Alemania/ $149 \mathrm{p}$.

FONTAGRO (FONDO REGIONAL DE TECNOLOGIA AGROPECUARIA). 2006. Charmetant Pierre. Diseminación por embriogénesis somática a gran escala en América Central y República Dominicana de variedades F1mejoradas de café arábica y de la variedad porta injerto (Nemaya) tolerantes a las principales enfermedades y plagas y de alta productividad

Hidalgo, M. 2007. Producción de híbrido F1 multiplicados por medio de cultivos in vitro en comparación a variedades comerciales en distintas regiones de Costa Rica.

Katzeff. P. 2001. El Manifestó de los Catadores de Café. Primera Edición. Managua, Nicaragua. 83p.

Lara, L. 2005. Efectos de la altitud, sombra, producción y fertilización sobre la calidad del café (Coffea Arabica L. Var. Caturra) producido en sistemas agroforestales de la zona cafetalera norcentral de Nicaragua. Tesis. Magíster Scientiae en Agroforestería Tropical CATIE. Turrialba, Costa Rica.pag 106.

Muscheler, R. 2006. Manejo de sombra para cafetales: Intensificación del la caficultora, menos sombras, mas insumo IN: El cafetal del futuro. (Eds). Pohlan. H, Soto, L y Barrrera, J. Shaker verlang Aachen. (2006) Pág. 39- 58.

1998. Tree- crop compatibility in agroforestry: Production and quality of coffea grown and managed tree shade in Costa Rica. Tesis Phd. Universidad of Florida. P. 219.

ORGANICOOP. 2007. Producción cafetalera sigue soportando una fuerte crisis. ( en linea). Managua, Ni. Consultado, 2 de junio.2008. Disponible en http://organicoop.blogspot.com/2007/09/caf-de-nicaragua.html.

Orozco, J. 2009. Significado de los resultados otorgados a las muestras de calidad organoléptica de los cultivares evaluados. (entrevista). Managua, CERCAFENIC. Comunicación Personal.

Pohlan. H, Soto, L y Barrera, J.2006. El Cafetal del futuro: retos y visiones. 1ed. Chiapas, México. Casa Editorial SHAKER. $462 \mathrm{p}$.

Santoyo, N; Escamilla, E, Robledo, JY Escamilla, E. 1996. Factores agronómicos y calidad del café. Confederación mexicana y productores de café. Universidad Autónoma Chapingo P (1- 7). 